\title{
Sentence Ordering with Manifold-based Classification in
}

\section{Multi-Document Summarization}

\author{
Paul D Ji \\ Centre for Linguistics and Philology \\ University of Oxford \\ paul_djilyahoo.co.uk
}

\begin{abstract}
In this paper, we propose a sentence ordering algorithm using a semi-supervised sentence classification and historical ordering strategy. The classification is based on the manifold structure underlying sentences, addressing the problem of limited labeled data. The historical ordering helps to ensure topic continuity and avoid topic bias. Experiments demonstrate that the method is effective.
\end{abstract}

\section{Introduction}

Sentence ordering has been a concern in text planning and concept-to-text generation (Reiter et al., 2000). Recently, it has also drawn attention in multi-document summarization (Barzilay et al., 2002; Lapata, 2003; Bollegala et al., 2005). Since summary sentences generally come from different sources in multi-document summarization, an optimal ordering is crucial to make summaries coherent and readable.

In general, the strategies for sentence ordering in multi-document summarization fall in two categories. One is chronological ordering (Barzilay et al., 2002; Bollegala et al., 2005), which is based on time-related features of the documents. However, such temporal features may be not available in all cases. Furthermore, temporal inference in texts is still a problem, in spite of some progress in automatic disambiguation of temporal information (Filatova

\author{
Stephen Pulman \\ Centre for Linguistics and Philology \\ University of Oxford \\ sgp@clg.ox.ac.uk
}

et al., 2001).

Another strategy is majority ordering (MO) (McKeown et al., 2001; Barzilay et al., 2002), in which each summary sentence is mapped to a theme, i.e., a set of similar sentences in the documents, and the order of these sentences determines that for summary sentences. To do that, a directed theme graph is built, in which if a theme $\mathrm{A}$ occurs behind another theme $\mathrm{B}$ in a document, B is linked to A no matter how far away they are located. However, this may lead to wrong theme correlations, since B's occurrence may rely on a third theme $\mathrm{C}$ and have nothing to do with A. In addition, when outputting theme orders, $\mathrm{MO}$ uses a kind of heuristic that chooses a theme based on its in-out edge difference in the directed theme graph. This may cause topic disruption, since the next choice may have no link with previous choices.

Lapata (2003) proposed a probabilistic ordering (PO) method for text structuring, which can be adapted to majority ordering if the training texts are those documents to be summarized. The primary evidence for the ordering are informative features of sentences, including words and their grammatical dependence relations, which needs reliable parsing of the text. Unlike in MO, selection of the next sentence here is based on the most recent one. However, this may lead to topic bias: i.e. too many sentences on the same topic.

In this paper, we propose a historical ordering (HO) strategy, in which the selection of the next sentence is based on the whole history of selection, not just the most recent choice. This 
strategy helps to ensure continuity of topics but to avoid topic bias at the same time.

To do that, we need to map summary sentences to those in documents. We formalize this as a kind of classification problem, with summary sentences as class labels. Since there are very few (only one) labeled examples for each class, we adopt a kind of semi-supervised classification method, which makes use of the manifold structure underlying the sentences to do the classification. A common assumption behind this learning paradigm is that the manifold structure among the data, revealed by higher density, provides a global comparison between data points (Szummer et al., 2001; Zhu et al., 2003; Zhou et al., 2003). Under such an assumption, even one labeled example is enough for classification, if only the structure is determined.

The remainder of the paper is organized as follows. In section 2, we give an overview of the proposed method. In section 3 5, we talk about the method including sentence networks, classification and ordering. In section 6 , we present experiments and evaluations. Finally in section 7, we give some conclusions and future work.

\section{Overview}

Fig. 1 gives the overall structure of the proposed method, which includes three modules: construction of sentence networks, sentence classification and sentence ordering.

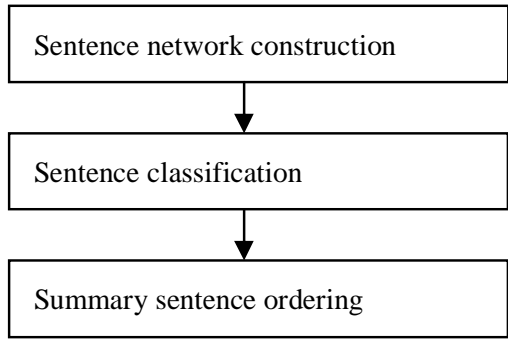

Fig. 1. Algorithm Overview

The first step is to build a sentence neighborhood network with weights on edges, which can serve as the basis for a Markov random walk (Tishby et al., 2000). The neighborhood is based on similarity between sentences, and weights on edges can be seen as transition probabilities for the random walk. From this network, we can derive new representations for sentences.

The second step is to make a classification of sentences, with each summary sentence as a class label. Since only one labeled example exists for each class, we use a semi-supervised method based on a Markov random walk to reveal the manifold structure for the classification.

The third step is to order summary sentences according to the original positions of their partners in the same class. During this process, the next selection of a sentence is based on the whole history of selection, i.e., the association of the sentence with all those already selected.

\section{Sentence Network Construction}

Suppose $S$ is the set of all sentences in the documents and a summary (a summary sentence may be not a document sentence), let $S=\left\{s_{1}\right.$, $\left.s_{2}, \ldots, s_{\mathrm{N}}\right\}$ with a distance metric $d\left(s_{\mathrm{i}}, s_{\mathrm{j}}\right)$, the distance between two sentences $s_{\mathrm{i}}$ and $s_{\mathrm{j}}$, which is based on the Jensen-Shannon divergence (Lin, 1991). We construct a graph with sentences as points by sorting the distances among the points in an ascending order and repeatedly connecting two points according to the order until a connected graph is obtained. Then, we assign a weight $w_{\mathrm{i}, \mathrm{j}}$, as in (1), to each edge based on the distance.

1) $w_{i, j}=\exp \left(-d\left(s_{i}, s_{j}\right) / \delta\right)$

The weights are symmetric, $w_{\mathrm{i}, \mathrm{i}}=1$ and $w_{\mathrm{i}, \mathrm{j}}=0$ for all non-neighbors ( $\delta$ is set as 0.6 in this work). 2 ) is the one-step transition probability $p\left(s_{\mathrm{i}}, s_{\mathrm{j}}\right)$ from $s_{\mathrm{i}}$ to $s_{\mathrm{j}}$ based on weights of neighbors.

2) $p\left(s_{i}, s_{j}\right)=\frac{w_{i, j}}{\sum_{k} w_{i, k}}$ 
Let $M$ be the $N \times N$ matrix and $M_{\mathrm{i}, \mathrm{j}}=p\left(s_{\mathrm{i}}, s_{\mathrm{j}}\right)$, then $\mathrm{M}^{\mathrm{t}}$ is the $t^{\text {th }}$ Markov random walk matrix, whose $i$, $j$-th entry is the probability $p_{\mathrm{t}}\left(s_{\mathrm{i}}, s_{\mathrm{j}}\right)$ of the transition from $s_{\mathrm{i}}$ to $s_{\mathrm{j}}$ after $t$ steps. In this way, each sentence $s_{\mathrm{j}}$ is associated with a vector of conditional probabilities $p_{\mathrm{t}}\left(s_{\mathrm{i}}, s_{\mathrm{j}}\right), i=1, \ldots, N$, which form a new manifold-based representation for $s_{\mathrm{j}}$. With such representations, sentences are close whenever they have a similar distribution over the starting points. Notice that the representations depend on the step parameter $t$ (Tishby et al., 2000). With smaller values of $t$, unlabeled points may be not connected with labeled ones; with bigger values of $t$, the points may be indistinguishable. So, an appropriate $t$ should be estimated.

\section{Sentence Classification}

Suppose $s_{1}, s_{2}, \ldots, s_{\mathrm{L}}$ are summary sentences and their labels are $c_{1}, c_{2}, \ldots, c_{\mathrm{L}}$ respectively. In our case, each summary sentence is assigned with a unique class label $c_{\mathrm{i}}, 1 \leq i \leq L$. This also means that for each class $c_{\mathrm{i}}$, there is only one labeled example, i.e., the summary sentence, $s_{\mathrm{i}}$.

Let $S=\left\{\left(s_{1}, c_{1}\right),\left(s_{2}, c_{2}\right), \ldots,\left(s_{\mathrm{L}}, c_{\mathrm{L}}\right), s_{\mathrm{L}+1}, \ldots, s_{\mathrm{N}}\right\}$, then the task of sentence classification is to infer the labels for unlabeled sentences, $s_{\mathrm{L}+1}, \ldots, s_{\mathrm{N}}$. Through the classification, we can get similar sentences for each summary sentence. To do that, we assume that each sentence has a distribution $p\left(c_{\mathrm{k}} \mid s_{\mathrm{i}}\right), 1 \leq k \leq L, 1 \leq i \leq N$, and these probabilities are to be estimated from the data.

Seeing a sentence as a sample from the $t$ step Markov random walk in the sentence graph, we have the following interpretation of $p\left(c_{\mathrm{k}} \mid s_{\mathrm{i}}\right)$.

$$
\text { 3) } p\left(c_{k} \mid s_{i}\right)=\sum_{j} p\left(c_{k} \mid s_{j}\right) p_{t}(j, i)
$$

This means that the probability of $s_{\mathrm{i}}$ belonging to $c_{\mathrm{k}}$ is dependent on the probabilities of those sentences belonging to $c_{\mathrm{k}}$ which will transit to $s_{\mathrm{i}}$ after $t$ steps and their transition probabilities.

With the conditional log-likelihood of labeled sentences 4 ) as the estimation criterion, we can use the EM algorithm to estimate $p\left(c_{\mathrm{k}} \mid s_{\mathrm{i}}\right)$, in which the E-step and M-step are 5) and 6) respectively.

4) $\sum_{k=1}^{L} \log p\left(c_{k} \mid s_{k}\right)=\sum_{k=1}^{L} \log \sum_{j=1}^{N} p\left(c_{k} \mid s_{j}\right) p_{t}(j, k)$

5) $p\left(s_{i} \mid s_{k}, c_{k}\right)=p\left(c_{k} \mid s_{i}\right) p_{t}(i, k) / \sum_{k=1}^{L} p\left(c_{k} \mid s_{i}\right) p_{t}(i, k)$

6) $p\left(c_{k} \mid s_{i}\right)=p\left(s_{i} \mid s_{k}, c_{k}\right) / \sum_{1 \leq k \leq L} p\left(s_{i} \mid s_{k}, c_{k}\right)$

The final class $c_{\mathrm{i}}$ for $s_{\mathrm{i}}$ is given in 7).

7) $c_{i}=\arg \max _{c_{k}} p\left(c_{k} \mid s_{i}\right)$

$p\left(c_{\mathrm{i}} \mid s_{\mathrm{i}}\right)$ is called the membership probability of $s_{\mathrm{i}}$. After classification, each sentence is assigned a label according to 7).

One key problem in this setting is to estimate the parameter $t$. A possible strategy for that is by cross validation, but it needs a large amount of labeled data. Here, following Szummer et al., 2001, we use marginal difference of probabilities of sentences falling in different classes as the estimation criterion, which is given in 8).

8) $m(S)=\sum_{s \in S}\left(L \max _{1 \leq k \leq L} p\left(c_{k} \mid s\right)-\sum_{1 \leq k \leq L} p\left(c_{k} \mid s\right)\right)$

To maximize 8), we can get an appropriate value for the parameter $t$, which means that a better $t$ should make sentences belong to some classes more prominently. Notice that the classes represented by summary sentences may be incomplete for all the sentences occurring in the documents, so some sentences will belong to the classes without obviously different probabilities. To avoid such sentences in the estimation of $t$, we only choose the top (40\%) sentences in a class based on their membership probabilities.

\section{Sentence Ordering}

After sentence classification, we get a class of similar sentences for each summary sentence, which is also a member of the class. With these sentence classes, we create a directed class graph based on the order of their member sentences in documents. In the graph, each sentence class is a 
node, and there exists a directed edge $e_{\mathrm{i}, \mathrm{j}}$ from one node $c_{\mathrm{i}}$ to another $c_{\mathrm{j}}$ if and only if there is $s_{\mathrm{i}}$ in $c_{\mathrm{i}}$ immediately appearing before $s_{\mathrm{j}}$ in $c_{\mathrm{j}}$ in the documents (the sentences not in classes are neglected). The weight of $e_{\mathrm{i}, \mathrm{j}}, F_{\mathrm{i}, \mathrm{j}}$, captures the frequency of such occurrence. We add one additional node denoting an initial class $c_{0}$, and it links to each class with a directed edge $e_{0, \mathrm{j}}$, the weight $F_{0, \mathrm{j}}$ of which is the frequency of the member sentences of the class appearing at the beginning of the documents.

Suppose the input is the class graph $G=\langle C, E\rangle$, where $C=\left\{c_{1}, c_{2}, \ldots, c_{\mathrm{L}}\right\}$ is the set of the classes, $E=\left\{e_{\mathrm{i}, \mathrm{j}} \mid 1 \leq \mathrm{i}, \mathrm{j} \leq L\right\}$ is the set of the directed edges, and $o$ is the ordering of the classes. Fig. 2 gives the ordering algorithm.

i) $c_{k} \leftarrow \max _{c_{i} \in C} F_{0, i}$

ii) $o \leftarrow o c_{\mathrm{k}}$

iii) For all $c_{\mathrm{i}}$ in $C, F_{0, i} \leftarrow F_{0, i}+F_{k, i}$

iv) Remove $c_{\mathrm{k}}$ from $C$ and $e_{\mathrm{k}, \mathrm{j}}$ and $e_{\mathrm{i}, \mathrm{k}}$ from $E$;

v) Repeat i)-iv) while $C \neq\left\{c_{0}\right\}$

vi) Return the order $o$.

Fig. 2 Ordering algorithm

In the algorithm, there are two main steps. Step i) selects the class whose member sentences occur most frequently immediately after those in $c_{0}$. Step iii) updates the weights of the edges $e_{0, \mathrm{i}}$. In fact, it can be seen as merge of the original $c_{0}$ and $c_{\mathrm{k}}$, and in this sense the updated $c_{0}$ represents the history of selections.

In contrast to the $\mathrm{MO}$ algorithm, the ordering algorithm here (HO) uses immediate back-front co-occurrence, while the MO algorithm uses relative back-front locations. On the other hand, the selection of a class is dependent on previous selections in $\mathrm{HO}$, while in $\mathrm{MO}$, the selection of a class is mainly dependent on its in-out edge difference.

In contrast to the $\mathrm{PO}$ algorithm, the selection of a class in $\mathrm{HO}$ is dependent on all previous selections, while in $\mathrm{PO}$, the selection is only related to the most recent one.

As an example, Fig. 3 gives an initial class graph. The output orderings by $\mathrm{PO}$ and $\mathrm{HO}$ are $\left[c_{1}, c_{3}, c_{4}, c_{2}\right]$ and $\left[c_{1}, c_{3}, c_{2}, c_{4}\right]$ respectively. The difference lies in whether to select $c_{4}$ or $c_{2}$ after selection of $c_{3}$. PO selects $c_{4}$ since it only considers the most recent selection, while $\mathrm{HO}$ selects $c_{2}$ because it considers all previous selections including $c_{1}$.

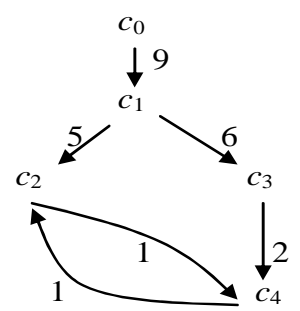

Fig. 3 Initial graph for PO and $\mathrm{HO}$

As another example, Fig. 4 gives the order of the classes in individual documents.

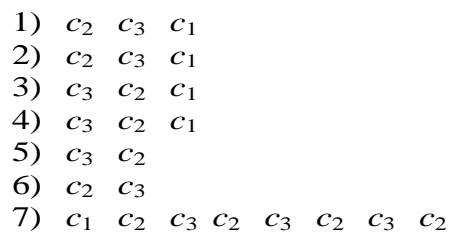

Fig. 4. Class orders in documents

From 1)-6), we can see some regularity among the order of the classes: $c_{2}$ and $c_{3}$ are interchangeable, while $c_{1}$ always appears behind $c_{2}$ or $c_{3}$. From 7), we can see that $c_{2}$ and $c_{3}$ still co-occur, while $c_{1}$ happens to occur at the beginning of the document. Thus, the appropriate ordering should be $\left[c_{2}, c_{3}, c_{1}\right]$ or $\left[c_{3}, c_{2}, c_{1}\right]$. Fig. 5 is the graph built by MO.

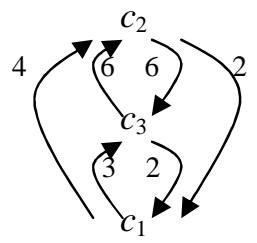

Fig. 5 Graph by MO 
According to $\mathrm{MO}$, the first node to be selected will be $c_{1}$, since the difference of its in-out edges $(+3)$ is bigger than that $(-2,-1)$ of other two nodes. Then the in-out edge differences for $c_{2}$ or $c_{3}$ are both 0 after removing edges associated with $c_{1}$, and either $c_{2}$ or $c_{3}$ will be selected. Thus, the output ordering should be $\left[c_{1}, c_{2}, c_{3}\right]$ or $\left[c_{1}, c_{3}, c_{2}\right]$.

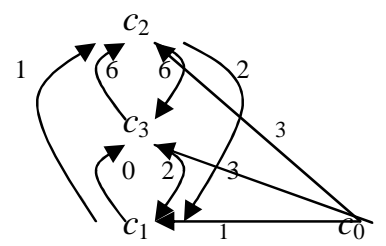

Fig. 6 Graph by $\mathrm{HO}$

Fig. 6 is the class graph built by HO. According to $\mathrm{HO}$, the first node to be selected will be $c_{2}$ or $c_{3}$, since $e_{0,1}=e_{0,2}=3>e_{0,1}=1$. Suppose $c_{2}$ is firstly selected, then $e_{0,3} \Leftarrow e_{0,3}+e_{2,3}=3+6=9$, while $e_{0,1} \Leftarrow$ $e_{0,1}+e_{2,1}=1+2=3$, so $c_{3}$ will be selected then. Finally the output ordering will be $\left[c_{2}, c_{3}, c_{1}\right]$. Similarly, if $c_{3}$ is firstly selected, the output ordering will be $\left[c_{3}, c_{2}, c_{1}\right]$.

\section{Experiments and Evaluation}

\subsection{Data}

We used the DUC04 document dataset. The dataset contains 50 document clusters and each cluster includes 20 content-related documents. For each cluster, 4 manual summaries are provided.

\subsection{Evaluation Measure}

The proposed method in this paper consists of two main steps: sentence classification and sentence ordering. For classification, we used pointwise entropy (Dash et al., 2000) to measure the quality of the classification result due to lack of enough labeled data. For a $n \times m$ matrix $M$, whose row vectors are normalized as 1 , its pointwise entropy is defined in 9).
9) $\left.E(M)=-\sum_{1<\leq \leq n \leq j \leq \leq n} \sum_{i, j} \log M_{i, j}+\left(1-M_{i, j}\right) \log \left(-M_{i, j}\right)\right)$

Intuitively, if $M_{\mathrm{i}, \mathrm{j}}$ is close to 0 or $1, E(M)$ tends towards 0 , which corresponds to clearer distinctions between classes; otherwise $E(M)$ tends towards 1 , which means there are no clear boundaries between classes. For comparison between different matrices, $E(M)$ needs to be averaged over $n \times m$.

For sentence ordering, we used Kendall's $\tau$ coefficient (Lapata, 2003), as defined in 10),

$$
\text { 10) } \tau=1-\frac{2\left(N_{I}\right)}{N(N-1) / 2}
$$

where, $N_{\mathrm{I}}$ is number of inversions of consecutive sentences needed to transform output of the algorithm to manual summaries. The measure ranges from -1 for inverse ranks to +1 for identical ranks, and can also be seen as a kind of edit similarity between two ranks: smaller values for lower similarity, and bigger values for higher similarity.

\subsection{Evaluation of Classification}

For sentence classification, we need to estimate the parameter $t$. We randomly chose 5 document clusters and one manual summary from the four. Fig. 7 shows the change of the average margin over all the top $40 \%$ sentences in a cluster with $t$ varying from 3 to 25 .

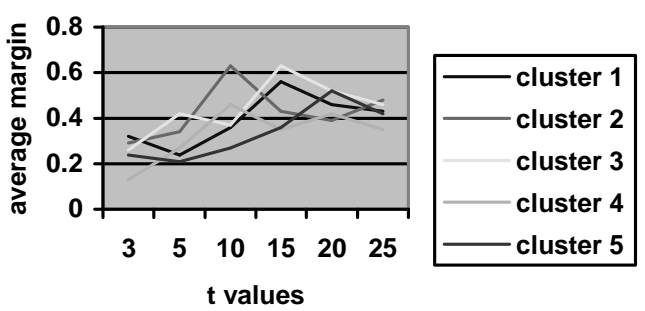

Fig. 7. Average margin and $t$

Fig. 7 indicates that the average margin changes with $t$ for each cluster and the values of $t$ maximizing the margin are different for different clusters. For the 5 clusters, the estimated $t$ is 16,8 , 14, 12 and 21 respectively. So we need to 
estimate the best $t$ for each cluster.

After estimation of $t$, EM was used to estimate the membership probabilities. Table 1 gives the average pointwise entropy for top $10 \%$ to top $100 \%$ sentences in each cluster, where sentences were ordered by their membership probabilities. The values were averaged over 20 runs, and for each run, 10 document clusters and one summary were randomly selected, and the entropy was averaged over the summaries.

\begin{tabular}{|l|l|l|l|}
\hline Sentences & E_Semi & E_SVM & Significance \\
\hline $10 \%$ & 0.23 & 0.22 & $\sim$ \\
\hline $20 \%$ & 0.26 & 0.27 & $\sim$ \\
\hline $30 \%$ & 0.32 & 0.43 & $*$ \\
\hline $40 \%$ & 0.35 & 0.49 & $* *$ \\
\hline $50 \%$ & 0.42 & 0.51 & $*$ \\
\hline $60 \%$ & 0.46 & 0.55 & $*$ \\
\hline $70 \%$ & 0.48 & 0.57 & $*$ \\
\hline $80 \%$ & 0.59 & 0.62 & $\sim$ \\
\hline $90 \%$ & 0.65 & 0.69 & $\sim$ \\
\hline $100 \%$ & 0.70 & 0.73 & $\sim$ \\
\hline
\end{tabular}

Table 1. Entropy of classification result

In Table 1, the column E_Semi shows entropies of the semi-supervised classification. It indicates that the entropy increases as more sentences are considered. This is not surprising since the sentences are ordered by their membership probabilities in a cluster, which can be seen as a kind of measure for closeness between sentences and cluster centroids, and the boundaries between clusters become dim with more sentences considered.

To compare the performance between this semi-supervised classification and a standard supervised method like Support Vector Machines (SVM), Table 1 also lists the average entropy of a SVM (E_SVM) over the runs. Similarly, we found that the entropy also increases as sentences increase. Table 2 also gives the significance sign over the runs, where $*, * *$ and $\sim$ represent p-values < $=0.01,(0.01,0.05]$ and $>0.05$, and indicate that the entropy of the semi-supervised classification is lower, significantly lower, or almost the same as that of SVM respectively.

Table 1 demonstrates that when the top $10 \%$ or $20 \%$ sentences are considered, the performance between the two algorithms shows no difference. The reason may be that these top sentences are closer to cluster centroids in both cases, and the cluster boundaries in both algorithms are clear in terms of these sentences.

For the top $30 \%$ sentences, the entropy for semi-supervised classification is lower than that for a SVM, and for the top $40 \%$, the difference becomes significantly lower. The reason may go to the substantial assumptions behind the two algorithms. SVM, based on local comparison, is successful only when more labeled data is available. With only one sentence labeled as in our case, the semi-supervised method, based on global distribution, makes use of a large amount of unlabeled data to reveal the underlying manifold structure. Thus, the performance is much better than that of a SVM when more sentences are considered.

For the top 50\% to 70\% sentences, E_Semi is still lower, but not by much. The reason may be that some noisy documents are starting to be included. For the top $80 \%$ to $100 \%$ sentences, the performance shows no difference again. The reason may be that the lower ranking sentences may belong to other classes than those represented by summary sentences, and with these sentences included, the cluster boundaries become unclear in both cases.

\subsection{Evaluation of Ordering}

We used the same classification results to test the performance of our ordering algorithm $\mathrm{HO}$ as well as MO and PO. Table 2 lists the Kendall's $\tau$ coefficient values for the three algorithms $\left(\tau_{-} 1\right)$. The value was averaged over 20 runs, and for each run, 10 summaries were randomly selected and the $\tau$ score was averaged over summaries. Since a summary sentence tends to generalize 
some sentences in the documents, we also tried to combine two or three consecutive sentences into one, and tested their ordering performance $\left(\tau \_2\right.$ and $\tau \_3$ ) respectively.

\begin{tabular}{|l|l|l|l|}
\hline$\tau$ & HO & MO & PO \\
\hline$\tau \_1$ & 0.42 & 0.31 & 0.33 \\
\hline$\tau \_2$ & 0.33 & 0.26 & 0.29 \\
\hline$\tau \_3$ & 0.27 & 0.21 & 0.25 \\
\hline
\end{tabular}

Table 2. $\tau$ scores for $\mathrm{HO}, \mathrm{MO}$ and $\mathrm{PO}$

Table 2 indicates that the combination of sentences harms the performance. To see why, we checked the classification results, and found that the pointwise entropies for two and three sentence combinations (for the top $40 \%$ sentence in each cluster) increase $12.4 \%$ and $18.2 \%$ respectively. This means that the cluster structure becomes less clear with two or three sentence combinations, which would lead to less similar sentences being clustered with summary sentences. This result also suggests that if the summary sentence subsumes multiple sentences in the documents, they tend to be not consecutive.

Fig. 8 shows change of $\tau$ scores with different number of sentences used for ordering, where $\mathrm{x}$ axis denotes top $(1-\mathrm{x})^{*} 100 \%$ sentences in each cluster. The score was averaged over 20 runs, and for each run, 10 summaries were randomly selected and evaluated.

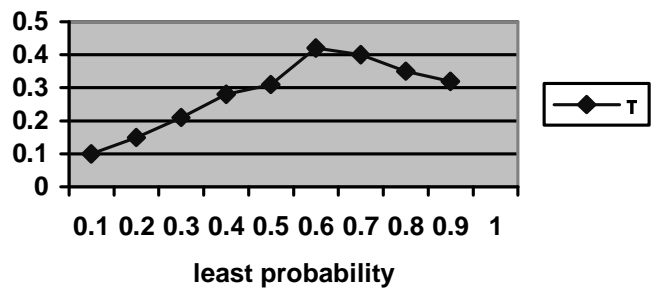

Fig. 8. $\tau$ scores and number of sentences

Fig. 8 indicates that with fewer sentences ( $x$ $>=0.7)$ used for ordering, the performance decreases. The reason may be that with fewer and fewer sentences used, the result is deficient training data for the ordering. On the other hand, with more sentences used $(\mathrm{x}<0.6)$, the performance also decreases. The reason may be that as more sentences are used, the noisy sentences could dominate the ordering. That's why we considered only the top $40 \%$ sentences in each cluster as training data for sentence reordering here.

As an example, the following is a summary for a cluster of documents about Central American storms, in which the ordering is given manually.

1) A category 5 storm, Hurricane Mitch roared across the northwest Caribbean with $180 \mathrm{mph}$ winds across a 350-mile front that devastated the mainland and islands of Central America.

2) Although the force of the storm diminished, at least 8,000 people died from wind, waves and flood damage.

3) The greatest losses were in Honduras where some 6,076 people perished.

4) Around 2,000 people were killed in Nicaragua, 239 in El Salvador, 194 in Guatemala, seven in Costa Rica and six in Mexico.

5) At least 569,000 people were homeless across Central America.

6) Aid was sent from many sources (European Union, the UN, US and Mexico).

7) Relief efforts are hampered by extensive damage.

Compared with the manual ordering, our algorithm $\mathrm{HO}$ outputs the ordering [1, 3, 4, 2, 5, 6, 7]. In contrast, $\mathrm{PO}$ and $\mathrm{MO}$ created the orderings $[1,3,4,5,6,7,2]$ and $[1,3,2,6,4,5,7]$ respectively. In HO's output, sentence 2 was put in the wrong position. To check why this was so, we found that sentences in cluster 2 and cluster 3 (clusters containing sentence 2 or sentence 3 ) were very similar, and the size of cluster 3 was bigger than that of cluster 2. Also we found that sentences in cluster 4 mostly followed those in cluster 3 . This may explain why the ordering $[1,3$, 4] occurred. Due to the link between cluster 2 and cluster 1 or 3 , sentence 2 followed sentence 4 in the ordering. In PO, sentence 2 was put at the end of the ordering, since it only considered the most recent selection when determining next, so cluster 1 would not be considered when determining the 
$4^{\text {th }}$ position. This suggests that consideration of selection history does in fact help to group those related sentences more closely, although sentence 2 was ranked lower than expected in the example.

In MO, we found sentence 2 was put immediately behind sentence 3 . The reason was that, after sentence 1 and 3 were selected, the in-edges of the node representing cluster 2 became 0 in the cluster directed graph, and its in-out edge difference became the biggest among all nodes in the graph, so it was chosen. For similar reasons, sentence 6 was put behind sentence 2. This suggests that it may be difficult to consider the selection history in MO, since its selection is mainly based on the current status of clusters.

\section{Conclusion and Future Work}

In this paper, we propose a sentence ordering method for multi-document summarization based on semi-supervised classification and historical ordering. For sentence classification, the semi-supervised classification groups sentences based on their global distribution, rather than on local comparisons. Thus, even with a small amount of labeled data (just 1 labeled example in our case) we nevertheless ensure good performance for sentence classification.

For sentence ordering, we propose a kind of history-based ordering strategy, which determines the next selection based on the whole selection history, rather than the most recent single selection in probabilistic ordering, which could result in topic bias, or in-out difference in MO, which could result in topic disruption.

In this work, we mainly use sentence-level information, including sentence similarity and sentence order, etc. In future, we may explore the role of term-level or word-level features, e.g., proper nouns, in the ordering of summary sentences. To make summaries more coherent and readable, we may also need to discover how to detect and control topic movement automatic summaries. One specific task is how to generate co-reference among sentences in summaries. In addition, we will also try other semi-supervised classification methods, and other evaluation metrics, etc.

\section{Reference}

Barzilay, R N. Elhadad, and K. McKeown. 2002. Inferring strategies for sentence ordering in multidocument news summarization. Journal of Artificial Intelligence Research, 17:35-55.

Bollegala D. Okazaki, N. Ishizuka, M. 2005. A Machine Learning Approach to Sentence Ordering for Multidocument Summarization, in Proceedings of IJCNLP.

Dash M. and H. Liu, (2000) Unsupervised feature selection, proceedings of PAKDD.

Filatova, E. \& Hovy, E. (2001) Assigning time-stamps to event-clauses. In Proceedings of AACL/EACL workshop on Temporal and Spatial Information Processing.

Lapata, M. 2003. Probabilistic text structuring: Experiments with sentence ordering. In Proceedings of the annual meeting of ACL 545-552.

Lin, J. 1991. Divergence Measures Based on the Shannon Entropy. IEEE Transactions on Information Theory, 37:1, 145-150.

McKeown K., Barzilay R. Evans D., Hatzivassiloglou V., Kan M., Schiffman B., \&Teufel, S. (2001). Columbia multi-document summarization: Approach and evaluation. In Proceedings of DUC.

Reiter, Ehud and Robert Dale. 2000. Building Natural Language Generation Systems. Cambridge University Press, Cambridge.

Szummer M. and T. Jaakkola. (2001) Partially labeled classification with markov random walks. NIPS14.

Tishby, N, Slonim, N. (2000) Data clustering by Markovian relaxation and the Information Bottleneck Method. NIPS 13.

Zhu, X., Ghahramani, Z., \& Lafferty, J. (2003) Semi-Supervised Learning Using Gaussian Fields and Harmonic Functions. ICML-2003.

Zhou D., Bousquet, O., Lal, T.N., Weston J. \& Schokopf B. (2003). Learning with local and Global Consistency. NIPS 16. pp: 321-328. 